\title{
TPE 核的螺旋手性
}

刘鸣华

国家纳米科学中心，北京 100190

\section{Helical Chirality of the TPE Core}

\section{LIU Minghua}

National Center for Nanoscience and Technology, Beijing 100190, P. R. China.

Email: liuminghua@nanoctr.cn

(a)

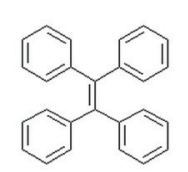

TPE

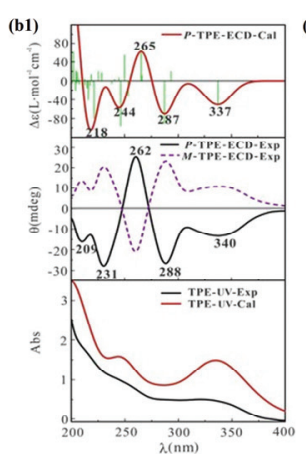

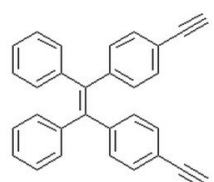

BETPE

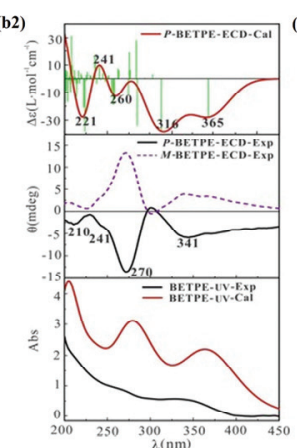

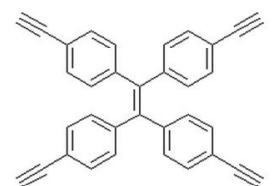

TETPE

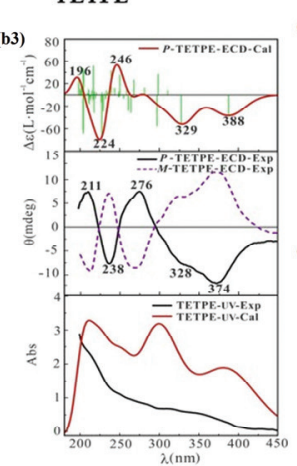

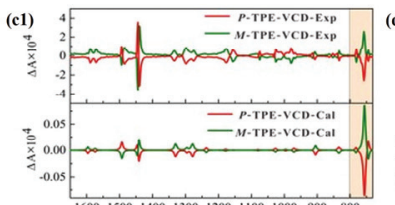

(c2)

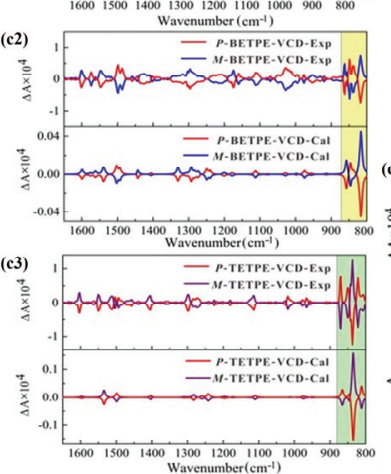

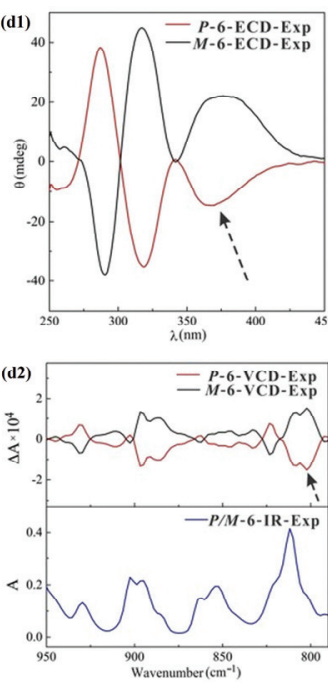

(a) TPE 及其衍生物。(b) 手性 TPE 及其衍生物的固体 ECD 和 UV 实验谱和计算谱。(c) 手性 TPE 及其衍生物 的固体 VCD 实验谱和计算谱。(d) 被有机框架锁住手性的 TPE 衍生物的固体 ECD 和 VCD-IR 实验谱

2001 年, 唐本忠等基于多苯环取代的噻咯衍 生物在良溶剂中不发光或发光微弱, 而在聚集状 态下苂光大大增强的现象, 率先提出了 “聚集诱 导发光(aggregation-induced emission, AIE)” 的概 念 ${ }^{1}$, 经过大量实验验证和理论模拟, 他们提出了 分子内运动受限是导致化合物产生 AIE 现象的主 要机理 ${ }^{2}$ 。AIE 化合物所具有的与传统有机发光材 料聚集猝灭发光 (aggregation-caused quenching, ACQ)现象的迥异秉性, 在聚集态或固态下较高的 发光效率, 理论上可提高光电器件效率和传感器 件灵敏度的优异性能等, 受到广泛关注 ${ }^{3}$ 。

四苯基乙烯(TPE)是典型的 AIE 化合物, 在聚
集态、固态或在特定的无机、有机和有机金属框架 中, 其连接多苯环的螺旋手性可以被固定(锁住), 从而可以观测到聚集诱导圆二色 (aggregationinduced $\mathrm{CD}, \mathrm{AICD}$ ) 和聚集诱导圆偏振发光 (aggregation-induced CPL, AICPL)等一系列手性 光谱性质 4,5 。这一类以 TPE 为核的化合物的绝对 构型的确定对于研究手性 AIE 聚合物或超分子螺 旋体系, 设计构建新型手性 AIE 功能材料都有重 要的意义。然而, 对于简单的 TPE-core 衍生物而 言, 精准确定其螺旋手性的绝对构型具有很大挑 战性, 尤其是对于没有重原子(只含有 $\mathrm{C} 、 \mathrm{H}$ )的化 合物, 即使采用先进的同步辐射等 X-射线单晶衍 
射技术，仍存在一定的局限性。

近期, 厦门大学章慧教授课题组与香港科技 大学唐本忠教授团队合作, 采用固体电子圆二色 (electronic circular dichroism, ECD)、振动圆二色 (vibrational circular dichroism, VCD) 实验谱结合晶 体结构分析和密度泛函理论(DFT)、含时密度泛函 理论(TDDFT)计算对四苯基乙烯及其衍生物的手 性立体化学特征进行了深入研究 6 。首次发现并提 出在 ECD 和 VCD 谱中均存在特征指纹区, 可用 于指认具有特征 TPE 核化合物的螺旋手性绝对构 型(构象) ${ }^{6}$ : (1) 若在 300-450 nm 波段内的第一个 $\mathrm{ECD}$ 吸收峰为正, TPE 核的螺旋手性为 $M$, 反之 则为 $P$ 构型; (2) 在 VCD 谱的低波数到高波数方 向, 若 730-850 $\mathrm{cm}^{-1}$ 区间的第一个最强 VCD 谱峰 的符号为正, 则 TPE 核螺旋手性为 $M$, 反之亦然。 此指纹特征区进一步被华中科技大学郑炎松教授 所提供的手性 TPE 衍生物样品 ${ }^{4}$ 的固体手性光谱 测试所证明。该研究所得规律或可以推广至含多 苯基的其它 AIE 化合物刚性发光核螺旋手性的研 究, 并且对设计合成具有强圆偏振发光性能的 AIE 分子提供有益借鉴。成果以 “Diagnostic Absolute Configuration Determination of Tetraphenylethene Core-Based Chiral Aggregation-Induced Emission Compounds: Particular Fingerprint Bands in Comprehensive Chiroptical Spectroscopy” 为题发表 于 The Journal of Physical Chemistry C ${ }^{6}$ 。

相关研究工作 7,8 始于 2010 年 9 月, 主要由 厦门大学、香港科技大学和华南理工大学的丁雷 博士、胡蓉蓉副教授、李丹博士、硕士生郭栋和博
士生藏启光接力完成。李丹进行了 $\mathrm{VCD}$ 光谱的测 试和理论计算工作并执笔写作, 手性光谱理论计 算得到山西大学王越奎教授的全面指导。

\section{References}

(1) Luo, J.; Xie, Z.; Lam, J. W. Y.; Cheng, L.; Chen, H.; Qiu, C.; Kwok, H. S.; Zhan, X.; Liu, Y.; Zhu, D.; Tang, B. Z. Chem. Commun. 2001, 37, 1740. doi: 10.1039/B105159H

(2) Mei, J.; Leung, N. L. C.; Kwok, R. T. K.; Lam, J. W. Y.; Tang, B. Z. Chem. Rev. 2015, 115, 11718. doi: 10.1021/acs.chemrev.5b00263

(3) Pan, L.; Luo, W.; Chen, M.; Liu, J.; Xu, L.; Hu, R.; Zhao, Z.; Qin, A.; Tang, B. Z. Chin. J. Org. Chem. 2016, 36, 1316. [潘凌翔, 罗文文, 陈明, 刘峻恺, 徐露, 胡蓉蓉, 赵祖金, 秦安军, 唐本忠. 有机化学, 2016, 36, 1316.] doi: 10.6023/cjoc201602020

(4) Xiong, J. B.; Feng, H. T.; Sun, J. P.; Xie, W. Z.; Yang, D.; Liu. M.; Zheng, Y. S. J. Am. Chem. Soc. 2016, 138, 11469. doi: $10.1021 /$ jacs.6b07087

(5) Qu, H.; Wang, Y.; Li, Z.; Wang, X. C.; Fang, H.; Tian, Z.; Cao, X. J. Am. Chem. Soc. 2017, doi: 10.1021/jacs.7b07657

(6) Li, D.; Hu, R.; Guo, D.; Zang, Q.; Li, J.; Wang, Y.; Tang, B. Z.; Zhang, H. J. Phys. Chem. C 2017, 121, 20947. doi: 10.1021/acs.jpcc.7b06679

(7) Ding, L.; Lin, L.; Liu, C.; Li, H.; Qin, A.; Liu, Y.; Song, L.; Zhang, H.; Tang, B. Z.; Zhao, Y. New J. Chem. 2011, 35, 1781. doi: $10.1039 / \mathrm{C} 1 \mathrm{NJ} 20185 \mathrm{~A}$

(8) Zhang, H.; Yan, J.; Wu, S.; Li, D.; Wan, S.; Ding, L.; Lin, L. Acta Phys. -Chim. Sin. 2013, 29, 2481. [章慧, 颜建新, 吴舒婷, 李丹, 万仕刚, 丁雷, 林丽榕. 物理化学学报, 2013, 29, 2481.] doi: 10.3866/PKU.WHXB201310152 